\title{
Nonunique Coincidence Point Results via Admissible Mappings
}

\author{
Erdal Karapınar $\mathbb{D},{ }^{1,2,3}$ Chi-Ming Chen, ${ }^{4}$ and Andreea Fulga $\mathbb{D}^{5}$ \\ ${ }^{1}$ Division of Applied Mathematics, Thu Dau Mot University, Binh Duong Province, Vietnam \\ ${ }^{2}$ Department of Mathematics, Çankaya University, 06790, Etimesgut, Ankara, Turkey \\ ${ }^{3}$ Department of Medical Research, China Medical University Hospital, China Medical University, 40402, Taichung, Taiwan \\ ${ }^{4}$ Institute for Computational and Modeling Science, National Tsing Hua University, Taiwan \\ ${ }^{5}$ Department of Mathematics and Computer Sciences, Transilvania University of Brasov, Brasov, Romania
}

Correspondence should be addressed to Andreea Fulga; afulga@unitbv.ro

Received 21 February 2021; Revised 12 March 2021; Accepted 15 March 2021; Published 29 March 2021

Academic Editor: Tuncer Acar

Copyright (C 2021 Erdal Karapınar et al. This is an open access article distributed under the Creative Commons Attribution License, which permits unrestricted use, distribution, and reproduction in any medium, provided the original work is properly cited.

\begin{abstract}
This paper is aimed at presenting some coincidence point results using admissible mapping in the framework of the partial $b$-metric spaces. Observed results of the article cover a number of existing works on the topic of "investigation of nonunique fixed points." We express an example to indicate the validity of the observed outcomes.
\end{abstract}

\section{Introduction and Preliminaries}

In 1974, Cirić [1] published the first paper on nonunique fixed point theory. Despite Banach's theorem, Ćirić [1] focused only on the existence of a fixed point, but not the uniqueness. The motivation of Ćirić [1] was inspired by Banach's motivation. As it is known, Banach's fixed point theorem is abstracted from Picard's paper, in which Picard [2] analyzed both the existence and uniqueness of the solution of the certain differential equation (see [3-5]). On the other hand, not all differential or integral equations have a unique solution. In the differential/integral equations, nonunique solutions are also crucial, for example, periodic solutions. Consequently, Ćirić [1] investigated the corresponding fixed point theorems that would be a tool in finding periodic solutions of the differential/integral equations. In the last five decades, a number of nonunique fixed point results have been reported in two ways: either proposing a new contraction type or changing the structure. The first example for the changing the contraction inequality, in the standard setup, was given by Achari [6] in 1976 and Pachpatte [7] in 1973. Fifteen years later, Ćirić and Jotić [8] proposed a new type of contraction inequalities in the context of complete metric space. This trend was followed by the attractive results
[9-13]. On the other hand side, in [14-17], the authors observed several characterizations of the unique fixed point results in the setting of complete $b$-metric spaces. Indeed, among the several extensions of metric structure, the true extension is the $b$-metric space. For this reason, observed nonunique fixed theorems in the context of $b$-metric space is very interesting and important, see also [18-20]. In addition, in [21-23], the characterization of fixed point theorems in partial metric spaces is crucial due to the potential application in the domain theory of computer science. Regarding the applied mathematics, nonunique fixed point results in cone metric spaces have taken attention [24].

In this paper, we consider a nonunique fixed point theorem in the context of the very general frame, partial $b$-metric spaces. An illustrative example is a set-up to indicate the validity of the main theorem.

Let $M$ be a nonempty set, a real number $\mathrm{s} \geq 1$, and $\mathbb{N}=$ $\{1,2,3, \cdots\}$.In this case, the triplet $\left(M, p_{b}, s\right)$ forms a partial $b$-metric space, on short $p_{b}$-ms.Undoubtedly, $b$-metric spaces (and ordinary metric spaces) are closely related to partial $b$ -metric spaces. Definitely, a $b$-metric space $(s \geq 1)$ is a partial $b$-metric space with zero self-distance and a partial metric space is a partial $b$-metric space with $s=1$. Moreover, a partial $b$-metric can define a $b$-metric space. Indeed, for example, 
let $p_{b}$ be a partial $b$-metric on $M$. Then, the functions $b_{p}^{\prime}, b_{p}$, $b_{p, m}: M \longrightarrow M$, where

$$
\begin{aligned}
b_{p}(u, v) & = \begin{cases}p_{b}(u, v), & \text { if } u \neq v, \\
0, & \text { if } u=v,\end{cases} \\
b_{p}^{\prime}(u, y) & =2 p_{b}(u, y)-p_{b}(u, u)-p_{b}(y, y), \\
b_{p, m}(u, y) & =p_{b}(u, y)-\min \left\{p_{b}(u, u), p_{b}(y, y)\right\}
\end{aligned}
$$

are $b$-metrics on $M$.

Definition 1. A function $p_{b}: M \times M \longrightarrow[0, \infty)$ is a partial $b$-metric on $M$ if for all $u, y, w \in M$, it satisfies the following conditions:

$$
\begin{aligned}
& \left(p_{b}\right)_{1} u=y \Longleftrightarrow p_{b}(u, u)=p_{b}(u, y)=p_{b}(y, y) \\
& \left(p_{b}\right)_{2} p_{b}(u, u) \leq p_{b} b(u, y) \\
& \left(p_{b}\right)_{3} p_{b}(u, y) \leq p(y, u) \\
& \left(p_{b}\right)_{4} p_{b}(u, y) \leq s\left[p_{b}(u, w)+p_{b}(w, u)\right]-p_{b}(w, w)
\end{aligned}
$$

Example 1. (see [25]). Let $p_{b}$ be a partial metric on the set $M$. Then, the functions $p_{b}: M \times M \longrightarrow[0, \infty)$ are given for all $u, y \in M$ by

(1) $p_{b}(u, y)=p(u, y)+b(u, y)$ is a partial $b$-metric on $M$ (where $b$ is a $b$-metric $(s>1)$ on $M$ )

(2) $p_{b}(u, y)=[p(u, y)]^{r}$ for $r \geq 1$, define a partial $b$-metrics on $M$ with coefficient $s=2^{r-1}$

Remark 2. From $(p b)_{1}$ and $(p b)_{2}$, it follows that if $u, y \in M$ are such that $p_{b}(u, y)=0$, then $u=y$.

Definition 3. (see $[26,27])$. Let $\left\{u_{n}\right\}$ be a sequence on the $\mathrm{p}_{b}-m s\left(M, \mathrm{p}_{b}, s \geq 1\right)$

(1) $\left\{u_{n}\right\}$ is $p_{b}$-convergent to $u \in M$ if $\lim _{n \rightarrow \infty} p_{b}\left(u, u_{n}\right)$ $=p_{b}(u, u)$

(2) $\left\{u_{n}\right\}$ is $p_{b}$-Cauchy if $\lim _{n, q \rightarrow \infty} p_{b}\left(\mathrm{u}_{\mathrm{n}}, \mathrm{u}_{\mathrm{q}}\right)$ exists and is finite

(3) $\left\{u_{n}\right\}$ is $0-p_{b}$-Cauchy if $\lim _{n, q \rightarrow \infty} p_{b}\left(u_{n}, u_{q}\right)=0$

(4) $\left(M, p_{b}, s \geq 1\right)$ is $p_{b}$-complete if every $p_{b}$-Cauchy sequence in $M$ is $p_{b}$-convergent

$$
\lim _{n, q \rightarrow \infty} p_{b}\left(u_{n}, u_{q}\right)=\lim _{n \rightarrow \infty} p_{b}\left(u_{n}, u\right)=p_{b}(u, u)
$$

(5) $\left(M, p_{b}, s \geq 1\right)$ is $0-p_{b}$-complete if every $0-p_{b}$-Cauchy sequence we can find $u \in M$ such that

$$
\lim _{n, q \rightarrow \infty} p_{b}\left(u_{n}, u_{q}\right)=\lim _{n \rightarrow \infty} p_{b}\left(u_{n}, u\right)=p_{b}(u, u)=0
$$

Moreover, in [26], the following interesting results were proved.

Lemma 4. (see [26]). Every $p_{b}$-complete $p_{b}$-ms $\left(M, p_{b}, s \geq 1\right)$ is 0 - $p_{b}$-complete.

Lemma 5. (see [26]). The $p_{b}-m s\left(M, p_{b}, s \geq 1\right)$ is 0 - $p_{b}$-complete if and only if the $b$-metric space $\left(M, b_{p}, s \geq 1\right)$ is complete, where the $b$-metric $b_{p}$ was defined in (3). hold.

They also showed that the converse affirmation does not

Let $R, S$ to self-mappings on the set $M$. We say that

(i) $S$ commutes with $R$ on $M$ if $R S u=S R u$ for all $u \in M$

(ii) a point $z \in M$ is a point of coincidence of $R$ and $S$ if we can find $u^{*} \in M$ such that $z=R u^{*}=S u^{*}$

(iii) a point $u^{*} \in M$ is a common fixed point of $R$ and $S$ if $R u^{*}=u^{*}=S u^{*}$

We will use the following notations:

$$
C_{c}(R, S)_{M}=\{u \in M \mid R u=S u\} M^{*}=M \backslash C_{c}(R, S)_{M} .
$$

In [28], the notion of $R$ - $\beta$-admissible mapping was introduced as follows:

(i) Let the function $\beta: M \times M \longrightarrow[0, \infty)$ and $R, S: M$ $\longrightarrow M$. The mapping $S$ is said to be $R$ - $\beta$-admissible if

$$
\beta(R u, R y) \geq 1 \text { implies } \beta(S u, S y) \geq 1,
$$

for all $u, y \in M$.

In case that $R=I_{M}$, the mapping $S$ is said to be $\beta$ -admissible.

Let $\left(M, p_{b}, s \geq 1\right)$ be a $p_{b}$-ms and $\beta ; M \times M \longrightarrow[0,+\infty)$. The space $M$ is $\beta$-regular if for every sequence $\left\{z_{n}\right\}$ in $M$ such that $z_{n} \longrightarrow z$ and $\beta\left(z_{n}, z_{n+1}\right) \geq 1$, there exists a subsequence $\left\{z_{n_{1}}\right\}$ of $\left\{z_{n}\right\}$ such that

$$
\beta\left(z_{n_{l}}, z_{*}\right) \geq 1,
$$

for all $l \in \mathbb{N}$.

Lemma 6. Let $R, S: M \longrightarrow M$ such that $S$ is a $R$ - $\beta$-admissible. If there exists $u_{0} \in M$ such that $\beta\left(R u_{0}, S u_{0}\right) \geq 1$, then

$$
\beta\left(R u_{n}, R u_{n+1}\right) \geq 1,
$$

where the sequence $\left\{u_{n}\right\}$ in $M$ is defined by $S u_{n}=R u_{n+1}$, for each $n \in \mathbb{N} \cup\{0\}$.

Proof. By the assumption $\beta\left(R u_{0}, S u_{0}\right) \geq 1$, since the mapping $S$ is $R$ - $\beta$-admissible, we get

$\beta\left(R u_{0}, R u_{1}\right)=\beta\left(R u_{0}, S u_{0}\right) \geq 1$ implies $\beta\left(R u_{1}, R u_{2}\right)=\beta\left(S u_{0}, S u_{1}\right) \geq 1$, 
and by induction, it follows that

$$
\beta\left(R u_{n}, R u_{n+1}\right) \geq 1
$$

for $n \in \mathbb{N} \cup\{0\}$.

\section{Main Results}

Following the idea in [29], we state the following results useful in the sequel.

Lemma 7. Let $\left(M, p_{b}, s \geq 1\right)$ be a $p_{b}$-ms. If $\left\{u_{n}\right\}$ is a sequence in $M$ such that there exists $\left\{z_{n}\right\}$ in $M$, satisfying the inequality

$$
p_{b}\left(u_{n}, u_{n+1}\right) \leq c p_{b}\left(u_{n-1}, u_{n}\right)
$$

for any $n \in \mathbb{N}$, then the sequence is $\left\{u_{n}\right\}$ and is $0-p_{b}$-Cauchy.

Proof. First of all, by (12), we get

$$
p_{b}\left(u_{n}, u_{n+1}\right) \leq c^{n} p_{b}\left(u_{0}, u_{1}\right)
$$

for all $n \in \mathbb{N}$. On the other hand, by using $(p b)_{4}$, we can derive that

$$
\begin{aligned}
p_{b}\left(u_{n}, u_{n+q}\right) \leq & s\left(p_{b}\left(u_{n}, u_{n+1}\right)\right. \\
& \left.+p_{b}\left(u_{n+1}, u_{n+q}\right)\right)-p_{b}\left(u_{n+1}, u_{n+1}\right) \\
\leq & s p_{b}\left(u_{n}, u_{n+1}\right) \\
& +s^{2}\left(p_{b}\left(u_{n+1}, u_{n+2}\right)+p_{b}\left(u_{n+2}, u_{n+2}, u_{n+q}\right)\right. \\
& --p_{b}\left(u_{n+1}, u_{n+1}\right)-p_{b}\left(u_{n+2}, u_{n+2}\right) \cdots \\
\leq & s p_{b}\left(u_{n}, u_{n+1}\right)+s^{2} p_{b}\left(u_{n+1}, u_{n+2}\right)+\cdots \\
& +s^{q} p_{b}\left(u_{n+q-1}, u_{n+q}\right)--\sum_{l=1}^{q-1} p_{b}\left(u_{n+l}, u_{n+l}\right) \\
\leq & s^{q}\left[p_{b}\left(u_{n}, u_{n+1}\right)+p_{b}\left(u_{n+1}, u_{n+2}\right)+\cdots\right. \\
& \left.+p_{b}\left(u_{n+q-1}, u_{n+q}\right)\right]--\sum_{l=1}^{q-1} p_{b}\left(u_{n+l}, u_{n+l}\right) .
\end{aligned}
$$

(1) If $c \in[0,1 / s)$, by (13) and (14), we get

$$
\begin{aligned}
p_{b}\left(u_{n}, u_{n+q}\right) & \leq \sum_{l=0}^{q-1} s^{l+1} c^{n+l} p_{b}\left(u_{0}, u_{1}\right)-\sum_{l=1}^{q-1} p_{b}\left(u_{n+l}, u_{n+l}\right) \\
& \leq s c^{n} \sum_{l=0}^{q-1}(s c)^{l} p_{b}\left(u_{0}, u_{1}\right) \\
& =s c^{n} \frac{1-(s c)^{q}}{1-s c} \longrightarrow 0 \text { as } n, q \longrightarrow \infty
\end{aligned}
$$

(2) If $c \in[1 / s, 1)$, thus $c^{n} \longrightarrow 0$ (as $\left.n \longrightarrow \infty\right)$. Moreover, there exits $l \in \mathbb{N}$ such that $c^{l}<1 / s$. This means $l>-$ $\log s / \log c$. Again, by (13) together with (14), we have

$$
\begin{aligned}
p_{b}\left(u_{n l}, u_{(n+1) l}\right) \leq & s^{l}\left[p_{b}\left(u n l, u_{n l+1}\right)+\cdots+p_{b}\left(u_{n l+l-1}, u_{(n+1) l}\right]\right. \\
& --\sum_{j=1}^{l-1} p_{b}\left(u_{n l+j}, u_{n l+j}\right) \\
\leq & s^{l} \sum_{j=0}^{l-1} c^{n l+j} p_{b}\left(u_{0}, u_{1}\right)-\sum_{j=1}^{l-1} p_{b}\left(u_{n l+j}, u_{n l+j}\right) \\
\leq & s^{l} c^{n l} \sum_{j=0}^{l-1} p_{b}\left(u_{0}, u_{1}\right) \\
& \leq c^{n l} \frac{s^{l} \cdot p_{b}\left(u_{0}, u_{1}\right)}{1-c} \longrightarrow 0 \text { as } n \longrightarrow \infty
\end{aligned}
$$

Thereby, letting $\lambda=c^{l}<1 / s$ by Case (i), we get that the sequence $\left\{u_{n l}\right\}$ is $0-p_{b}$-Cauchy sequence, which means that

$$
\lim _{n, q \rightarrow \infty} p_{b}\left(u_{n l}, u_{q l}\right)=0
$$

On the other hand,

$$
\begin{aligned}
p_{b}\left(u_{l[n / l]}, u_{n}\right) \leq & s\left(p_{b}\left(u_{l[n / l]}, u_{l[n / l]+1}\right)\right. \\
& \left.+p_{b}\left(u_{l[n / l]+1}, u_{n}\right)\right)-p_{b}\left(u_{l[n / l]+1}, u_{l[n / l]+1}\right) \\
\leq & s^{l}\left[p_{b}\left(u_{l[n / l]}, u_{l[n / l]+1}\right)+\cdots+p_{b}\left(u_{n-1}, u_{n}\right)\right] \\
& --\left(p_{b}\left(u_{l[n / l]+1}, u_{l[n / l]+1}\right)+\cdots+p_{b}\left(u_{n-1}, u_{n-1}\right)\right),
\end{aligned}
$$

and using (13), we have

$$
\begin{aligned}
p_{b}\left(u_{l[n / l]}, u_{n}\right) & \leq s^{l}\left[c^{l[n / l]}+\cdots+c^{n-1}\right] p_{b}\left(u_{0}, u_{1}\right) \\
& \leq s^{l} c^{l[n / l]} \frac{p_{b}\left(u_{0}, u_{1}\right)}{1-c} \longrightarrow 0 \text { as } n \longrightarrow \infty
\end{aligned}
$$

Finally, combining relations (19) and (17) and keeping in mind $(p b)_{4}$, we have

$$
\begin{aligned}
p_{b}\left(u_{n}, u_{q}\right) \leq & {\left[p_{b}\left(u_{n}, u_{l[n / l]}\right)\right.} \\
& \left.+p_{b}\left(u_{l[n / l]}, u_{q}\right)\right]-p_{b}\left(u_{[[n / l]}, u_{l[n / l]}\right) \\
\leq & s p_{b}\left(u_{n}, u_{l[n / l]}\right) \\
& +s^{2} p_{b}\left(u_{l[n / l]}, u_{l[q / l]}\right)+s^{2} p_{b}\left(u_{l[q / l]}, u_{q}\right) \\
& --p_{b}\left(u_{l[n / l]}, u_{l[n / l]}\right)-p_{b}\left(u_{l[q / l]}, u_{l[q / l]}\right) \\
\leq & s p_{b}\left(u_{n}, u_{l[n / l]}\right) \\
& +s^{2} p_{b}\left(u_{l[n / l]}, u_{l[q / l]}\right)+s^{2} p_{b}\left(u_{l[q / l]}, u_{q}\right) \longrightarrow 0 \text { as } n, q \longrightarrow \infty
\end{aligned}
$$

Thereupon, the sequence $\left\{u_{n}\right\}$ is $0-p_{b}$-Cauchy.

Therefore, $\left\{u_{n}\right\}$ is a $0-p_{b}$-Cauchy sequence. 
Theorem 8. Let $\left(M, p_{b}, s \geq 1\right)$ be a complete $p_{b}$-ms and two mappings $R, S: M \longrightarrow M$. Suppose that there exists $\kappa \in(0,1)$ such that

$$
\begin{gathered}
\beta(R u, R y) \min \left\{p_{b}(S u, S y), p_{b}(S y, R y)\right\} \\
--\min \left\{b_{p}(S u, R y), b_{p}(S y, R u)\right\} \\
\leq \kappa \max \left\{p_{b}(R u, R y), p_{b}(S u, R u)\right\},
\end{gathered}
$$

for all $u, y \in M$, such that $u \neq y$ when $u, y \in C_{c}(R, S)_{M}$. Suppose also that

(a) $S(M) \subset R(M)$ and $\left(R(M), p_{b}, s\right)$ is a 0 - $p_{b}$-complete $p_{b}-m s$

(b) $S$ is $R-\beta$-admissible, and there exists $u_{0} \in M$ such that $\beta\left(R u_{0}, S u_{0}\right) \geq 1$

(c) $M$ is $\beta$-regular

Then, the mappings $S$ and $R$ have a point of coincidence.

Proof. Let $u_{0}$ be an arbitrary point in $M$, such that $\beta\left(R u_{0}, S\right.$ $\left.u_{0}\right) \geq 1$. Thus, since $S(M) \subset R(M)$, there exists $u_{1} \in M$ such that $S u_{0}=R u_{1}$. Thereupon, $S u_{1} \in S(M) \subset R(M)$ and we can find $u_{2} \in M$ such that $S u_{1}=R u_{2}$. In this way, we can build a sequence $\left\{u_{n}\right\} \subseteq M$ as follows:

having defined $u_{n} \in M$, we let $u_{n+1} \in M$ such that $S u_{n}=R u_{n+1}$,

for all $n \in \mathbb{N} \cup\{0\}$. Letting $u=u_{n}$ and $y=u_{n+1}$ in (ref1T1) and taking into account Lemma 6 , we have

$$
\begin{aligned}
\min & \left\{p_{b}\left(S u_{n}, S u_{n+1}\right), p_{b}\left(S u_{n+1}, R u_{n+1}\right)\right\} \\
& \quad-\min \left\{b_{p, m}\left(S u_{n}, R u_{n+1}\right), b_{p, m}\left(S u_{n+1}, R u_{n}\right)\right\} \\
\leq & \beta\left(R u_{n}, R u_{n+1}\right) \min \left\{p_{b}\left(S u_{n}, S u_{n+1}\right), p_{b}\left(S u_{n+1}, R u_{n+1}\right)\right\} \\
& \quad-\min \left\{b_{p}\left(S u_{n+1}, R u_{n}\right)\right\} \\
\leq & \kappa \max \left\{p_{b}\left(R u_{n}, R u_{n+1}\right), p_{b}\left(S u_{n}, R u_{n}\right)\right\} .
\end{aligned}
$$

Keeping in mind (22), we get

$$
\begin{aligned}
\min & \left\{p_{b}\left(R u_{n+1}, R u_{n+2}\right), p_{b}\left(R u_{n+1}, R u_{n}\right), p_{b}\left(R u_{n+2}, R u_{n+1}\right)\right\} \\
& --\min \left\{b_{p}\left(R u_{n+1}, R u_{n+1}\right), b_{p}\left(R u_{n+2}, R u_{n}\right)\right\} \\
\leq & \kappa \max \left\{p_{b}\left(R u_{n}, R u_{n+1}\right), p_{b}\left(R u_{n+1}, R\left(u_{n}\right)\right)\right\} \\
= & \kappa p_{b}\left(R u_{n}, R u_{n+1}\right),
\end{aligned}
$$

which is equivalent with

$$
\begin{aligned}
\min & \left\{p_{b}\left(R u_{n+1}, R u_{n+2}\right), p_{b}\left(R u_{n+1}, R u_{n}\right)\right\} \\
& \quad--\min \left\{b_{p}\left(R u_{n+1}, R u_{n+1}\right), b_{p}\left(R u_{n+2}, R u_{n}\right)\right\} \\
\leq & \kappa p_{b}\left(R u_{n}, R u_{n+1}\right) .
\end{aligned}
$$

Therefore, we get

$$
p_{b}\left(R u_{n+1}, R u_{n+2}\right) \leq \kappa p_{b}\left(R u_{n}, R u_{n+1}\right),
$$

for any $n \in \mathbb{N} \cup\{0\}$.Let now $\left\{z_{n}\right\}$ be a sequence in $M$, with $z_{n}=R u_{n+1}=S u_{n}, n \in \mathbb{N} \cup\{0\}$. First of all, we mention that $z_{n} \neq z_{n+1}$ for every $n \in \mathbb{N}$. Indeed, if we suppose that there exists $m_{0} \in \mathbb{N} \cup\{0\}$ such that $z_{m_{0}}=z_{m_{0}+1}$, thus by (22), we have

$$
R u_{m_{0}+1}=S u_{m_{0}}=z_{m_{0}}=z_{m_{0}+1}=S u_{m_{0}+1}
$$

so that $z_{m_{0}+1}$ is a point of coincidence. Thus, $z_{n} \neq z_{n+1}$ for every $\mathbb{N} \cup\{0\}$ and (28) can be rewritten as

$$
p_{b}\left(z_{n}, z_{n+1}\right) \leq \kappa p_{b}\left(z_{n-1}, z_{n}\right) .
$$

Therefore, according to Lemma 7 , the sequence $\left\{z_{n}\right\}$ is 0 $-p_{b}$-Cauchy. Since the space is $0-p_{b}$-complete, it follows that there is $z \in M$ such that

$$
\lim _{n, q \rightarrow \infty} p_{b}\left(z_{n}, z_{q}\right)=\lim _{n \rightarrow \infty} p_{b}\left(z_{n}, z\right)=\mathrm{p}_{b}(z, z)=0
$$

But, on the other hand, since $z_{n}=R u_{n+1}$ and the space $\left(R(M), p_{b}, s\right)$ is $0-p_{b}$-complete, we can find $u_{*} \in M$, with $z=R u_{*}$. Thus,

$$
\lim _{n \rightarrow \infty} p_{b}\left(S u_{n}, R u_{*}\right)=\lim _{n \rightarrow \infty} p_{b}\left(R u_{n}, R u_{*}\right)=p_{b}\left(R u_{*}, R u_{*}\right)=0
$$

Supposing that $R u_{*} \neq S u_{*}$ for $u=u_{n_{l}}$ and $y=u_{*}$ and taking into account the $\beta$-regularity of the space $M$, we have

$$
\begin{aligned}
\min & \left\{p_{b}\left(S u_{n_{l}}, S u_{*}\right), p_{b}\left(S u_{*}, R u_{*}\right)\right\} \\
& -\min \left\{b_{p}\left(S u_{n_{l}}, R u_{*}\right), b_{p}\left(S u_{*}, R u_{n_{l}}\right)\right\} \\
\leq & \leq \beta\left(z_{n_{l}}, z\right) \min \left\{p_{b}\left(S u_{n_{l}}, S u_{*}\right), p_{b}\left(S u_{*}, R u_{*}\right)\right\} \\
& -\min \left\{b_{p}\left(S u_{n_{l}}, R u_{*}\right), b_{p}\left(S u_{*}, R u_{n_{l}}\right)\right\} \\
= & \beta\left(R u_{n_{l}}, R u_{*}\right) \min \left\{p_{b}\left(S u_{n_{l}}, S u_{*}\right), p_{b}\left(S u_{*}, R u_{*}\right)\right\} \\
& -\min \left\{b_{p}\left(S u_{n_{l}}, R u_{*}\right), b_{p}\left(S u_{*}, R u_{n_{l}}\right)\right\} \\
\leq & \kappa \max \left\{p_{b}\left(R u_{n_{l}}, R u_{*}\right), p_{b}\left(S u_{n_{l}}, R u_{n_{l}}\right)\right\} .
\end{aligned}
$$

If $\min \left\{p_{b}\left(S u_{n_{l}}, S u_{*}\right), p_{b}\left(S u_{*}, R u_{*}\right)\right\}=p_{b}\left(S u_{*}, R u_{*}\right)$, the above inequality becomes

$$
\begin{gathered}
p_{b}\left(S u_{*}, R u_{*}\right)-\min \left\{p_{b}\left(S u_{n_{l}}, S u_{*}\right), p_{b}\left(S u_{*}, R u_{*}\right)\right\} \\
\quad-\min \left\{b_{p}\left(S u_{n_{l}}, R u_{*}\right), b_{p}\left(S u_{*}, R u_{n_{l}}\right)\right\} \\
\leq \kappa \max \left\{p_{b}\left(R u_{n_{l}}, R u_{*}\right), p_{b}\left(S u_{n_{l}}, R u_{n_{l}}\right)\right\} .
\end{gathered}
$$

Letting $l \longrightarrow \infty$ and taking into account (28) and (30), we get

$$
p_{b}\left(S u_{*}, R u_{*}\right)=0 \text {, }
$$


and by $(p b)_{1},(p b)_{1}$, we have $S u_{*}=R u_{*}$.If $\min \left\{p_{b}\left(S u_{n_{l}}, S u_{*}\right)\right.$, $\left.p_{b}\left(S u_{*}, R u_{*}\right)\right\}=p_{b}\left(S u_{n_{l}}, S u_{*}\right)$, we find that $\lim _{l \rightarrow \infty} p_{b}\left(S u_{n_{l}}\right.$, $\left.S u_{*}\right)=0$. On the other hand, by $(p b)_{4}$,

$$
p_{b}\left(S u_{*}, R u_{*}\right) \leq s\left[p_{b}\left(S u_{*}, S u_{n_{l}}\right)+p_{b}\left(S\left(u_{n_{l}}, R u_{*}\right)\right]-p_{b}\left(S\left(u_{n_{l}}, S u_{n_{l}}\right),\right.\right.
$$

and then, $p_{b}\left(S u_{*}, R u_{*}\right)=0$, as $l \longrightarrow \infty$. This proves that $z$ $=S u_{*}=R u_{*}$, that is, $z$ is a point of coincidence for $S$ and $R$.

Example 2. Let $M=[0, \infty)$ and $p_{b}: M \times M \longrightarrow[0, \infty)$ be a partial $b$-metric, where $p_{b}(u, y)=(\max \{u, y\})^{2}$. Let the mappings $S, R: M \longrightarrow M$,

$$
\begin{aligned}
& S u= \begin{cases}\frac{u+1}{2}, & \text { if } u \in[0,1], \\
3, & \text { if } u>1,\end{cases} \\
& R u= \begin{cases}\frac{u+2}{4}, & \text { if } u \in[0,1], \\
\frac{u+5}{10}, & \text { if } u>1,\end{cases}
\end{aligned}
$$

and the function $\beta: M \times M \longrightarrow[0, \infty)$,

$$
\beta(x, v)= \begin{cases}2, & \text { for } x=v=\frac{1}{2} \\ 3, & \text { for } x=v=3 \\ 1, & \text { for } x, v \geq 4 \\ 0, & \text { otherwise }\end{cases}
$$

Obviously, since $x=R u \geq 4$ for $u \geq 35$ we have

(i) For $u, y \geq 35$

$$
\begin{array}{r}
\beta(R u, R y)=1 \Longrightarrow \beta(S u, S v)=\beta(3,3)=3>1, \\
\beta\left(\frac{1}{2}, \frac{1}{2}\right)=\beta(R(0), R(0))=2 \Longrightarrow \beta(S(0), S(0))=\beta\left(\frac{1}{2}, \frac{1}{2}\right)=2, \\
\beta(3,3)=\beta(R(25), R(25))=3 \Longrightarrow \beta(S(25), S(25))=\beta(3,3)=3 .
\end{array}
$$

Moreover,

$$
\begin{aligned}
& \beta(R u, R y) \min \left\{p_{b}(S u, S y), p_{b} b(S y, R y)\right\} \\
& \quad--\min \left\{b_{p}(S u, R y), b_{p}(S y, R u)\right\} \\
& \quad \leq \min \left\{p_{b}(3,3), p_{b}(3, R y\}\right. \\
& \quad=9, \leq \kappa \cdot 16 \leq \kappa \cdot \max \left\{p_{b}(R u, R y), p_{b}(S u, R u)\right\},
\end{aligned}
$$

for any $9 / 16<\kappa<1$. (ii) All other cases are uninteresting due to the way the function $\beta$ was defined

Consequently, by Theorem 8 , the mappings $S, R$ have points of coincidence. These are $1 / 2=S(0)=R(0)$, respectively, $3=S(25)=R(25)$.

Corollary 9. Let $\left(M, p_{b}, s \geq 1\right)$ be a complete $p_{b}$-ms and two mappings $R, S: M \longrightarrow M$. Suppose that there exists $\kappa \in(0,1)$ such that

$$
\begin{aligned}
\min & \left\{p_{b}(S u, S y), p_{b}(S y, R y)\right\} \\
& --\min \left\{b_{p}(S u, R y), b_{p}(S y, R u)\right\} \\
\leq & \kappa \max \left\{p_{b}(R u, R v), p_{b}(S u, R u)\right\},
\end{aligned}
$$

for every $u, y \in M$, such that $u \neq y$ when $u, y \in C_{c}(R, S)_{M}$. If $S$ $(M) \subset R(M)$ and $\left(R(M), p_{b}, s\right)$ is a $0-p_{b}$-complete $p_{b}$-ms, then the mappings $S$ and $R$ have a point of coincidence.

Proof. It is enough to choose $\beta(u, y)=1$ in Theorem 8 .

Theorem 10. Let $\left(M, p_{b}, s \geq 1\right)$ be a complete $p_{b}$-ms and $a$ mapping $S: M \longrightarrow M$. Suppose that there exists $\kappa \in(0,1)$ such that

$$
\begin{gathered}
\beta(u, y) \min \left\{p_{b}(S u, S y), p_{b}(S y, y)\right\} \\
--\min \left\{b_{p}(S u, y), b_{p}(S y, u)\right\} \\
\leq \kappa \max \left\{p_{b}(u, y), p_{b}(S u, u)\right\}
\end{gathered}
$$

for every $u, y \in M$, such that $u \neq y$. Suppose also that

(a) $S$ is $\beta$-admissible, and there exists $u_{0} \in M$ such that $\beta$ $\left(u_{0}, S u_{0}\right) \geq 1$

(b) $M$ is $\beta$-regular

Then, the mapping S has a fixed point.

Proof. Put $R=I_{M}$ in Theorem 8 .

Corollary 11. Let $\left(M, p_{b}, s \geq 1\right)$ be a complete $p_{b}$-ms and $a$ mapping $S: M \longrightarrow M$. Suppose that there exists $\kappa \in(0,1)$ such that

$$
\begin{aligned}
\min & \left\{p_{b}(S u, S y), p_{b}(S y, y)\right\} \\
& --\min \left\{b_{p}(S u, y), b_{p}(S y, u)\right\} \\
\leq & \kappa \max \left\{p_{b}(u, y), p_{b}(S u, u)\right\},
\end{aligned}
$$

for every $u, y \in M, u \neq y$. Then, the mapping S has a fixed point.

Proof. It is enough to choose $\beta(u, y)=1$ in Theorem 10 .

Theorem 12. Let $\left(M, p_{b}, s \geq 1\right)$ be a complete $p_{b}$-ms and two mappings $R, S: M \longrightarrow M$. Suppose that there exist $\kappa \in(0,1)$ 
and $a>0$ such that

$$
\beta(R u, R y) M_{S}^{1}(u, y)-a \cdot N_{S, R}^{1}(u, y) \leq \kappa p_{b}(S u, R u) p_{b}(S y, R y),
$$

where

$M_{S, R}^{1}(u, y)=\min \left\{\left[p_{b}(S u, S y)\right]^{2},\left[p_{b}(S y, R y)\right]^{2}\right\}$,

$N_{S, R}^{1}(u, y)=\min \left\{b_{p}(S u, R y) b_{p}(S y, R u), p_{b}(S u, R y) p_{b}(S u, S y), p_{b}(S y, R u) p_{b}(R u, R y)\right\}$,

for every $u, y \in M$, such that $u \neq y$ when $u, y \in C_{c}(R, S)_{M}$. Suppose also that:

(a) $S(M) \subset R(M)$ and $\left(R(M), p_{b}, s\right)$ is a 0 - $p_{b}$-complete $p_{b}-m s$ (b) $S$ is $R$ - $\beta$-admissible, and there exists $u_{0} \in M$ such that $\beta\left(R u_{0}, S u_{0}\right) \geq 1$

(c) $M$ is $\beta$-regular

Then, the mappings $S$ and $R$ have a point of coincidence.

Proof. Starting with a point $u_{0} \in M$ such that $\beta\left(R u_{0}, S u_{0}\right) \geq 1$, we build the sequences $\left\{u_{n}\right\},\left\{z_{n}\right\}$ as in Theorem 8 ,

$$
z_{n}=R u_{n+1}=S u_{n}, \text { for all } n \in \mathbb{N}
$$

Using the same arguments, we can assume that $z_{n} \neq z_{n+1}$, also, for all $n \in \mathbb{N}$. Thus, for $u=u_{n}, y=u_{n+1}$,

$$
\begin{aligned}
M_{S, R}^{1}\left(u_{n}, u_{n+1}\right) & =\min \left\{\left[p_{b}\left(S u_{n}, S u_{n+1}\right)\right]^{2},\left[p_{b}\left(S u_{n+1}, R u_{n+1}\right)\right]^{2}\right\} \\
& \left.=\min \left\{p_{b}\left(z_{n}, z_{n+1}\right)\right]^{2},\left[p_{b}\left(z_{n+1}, z_{n}\right)\right]^{2}\right\} \\
& =\left[p_{b}\left(z_{n+1}, z_{n}\right)\right]^{2}, \\
N_{S, R}^{1}\left(u_{n}, u_{n+1}\right) & =\min \left\{\begin{array}{c}
b_{p}\left(S u_{n}, R u_{n+1}\right) b_{p}\left(S u_{n+1}, R u_{n}\right), p_{b}\left(S u_{n}, R u_{n+1}\right) p_{b}\left(S u_{n}, S u_{n+1}\right), \\
p_{b}\left(S u_{n+1}, R u_{n}\right) p_{b}\left(R u_{n}, R u_{n+1}\right)
\end{array}\right\} \\
& =\min \left\{\begin{array}{c}
b_{p}\left(z_{n}, z_{n}\right) b_{p}\left(z_{n+1}, z_{n-1}\right), p_{b}\left(z_{n}, z_{n+1}\right) p_{b}\left(z_{n}, z_{n+1}\right), \\
p_{b}\left(z_{n+1}, u_{n-1}\right) p_{b}\left(u_{n-1}, z_{n}\right)
\end{array}\right\}=0,
\end{aligned}
$$

and taking into account Lemma 6, (42) becomes

$$
\begin{aligned}
M_{S, R}^{1}\left(u_{n}, u_{n+1}\right) & \leq \beta\left(R u_{n}, R u_{n+1}\right) M_{S, R}^{1}\left(u_{n}, u_{n+1}\right)-a \cdot N_{S, R}^{1}\left(u_{n}, u_{n+1}\right) \\
& \leq \kappa p_{b}\left(S u_{n}, R u_{n}\right) \cdot p_{b}\left(S u_{n+1}, R u_{n+1}\right) .
\end{aligned}
$$

Taking into account (46), the above inequality turns into

$$
\left[p_{b}\left(z_{n}, z_{n+1}\right)\right]^{2} \leq \kappa p_{b}\left(z_{n}, z_{n-1}\right) p_{b}\left(z_{n+1}, z_{n}\right)
$$

or equivalent (since $z_{n} \neq z_{n+1}$ )

$$
p_{b}\left(z_{n}, z_{n+1}\right) \leq \kappa p_{b}\left(z_{n}, z_{n-1}\right)
$$

Accordingly, from Lemma 7, it follows that the sequence $\left\{z_{n}\right\}$ is $0-p_{b}$-Cauchy and due to the completeness of the space, there exists $z \in M$ such that $\left.\lim n \longrightarrow \infty \mathrm{p}_{b}\left(z_{n}\right), z\right)$ $=p_{b}(z, z)=0$. Following the corresponding lines in Theorem 8 , we can find $u_{*} \in M$ such that $R u_{*}=z$. Supposing that $R u_{*} \neq S u^{*}$ for $u=u_{n_{l}}$ and $\mathrm{y}=\mathrm{u}_{*}$ and taking into account the assumption (c),

$$
\begin{aligned}
M_{S, R}^{1}\left(u_{n_{l}}, u_{*}\right) & \leq \beta\left(R u_{n_{l}}, R u_{*}\right) M_{S, R}^{1}\left(u_{n_{l}}, u_{*}\right)-a \cdot N_{S, R}^{1}\left(u_{n_{l}}, u_{*}\right) \\
& \leq \kappa p_{b}\left(S u_{n_{l}}, R u_{n_{l}}\right) \cdot p_{b}\left(S u_{*}, R u_{*}\right),
\end{aligned}
$$

where

$$
\begin{aligned}
& M_{S, R}^{1}\left(u_{n_{l}}, u_{*}\right)=\min \left\{\left[p_{b}\left(S u_{n_{l}}, S u_{*}\right)\right]^{2},\left[p_{b}\left(S u_{*}, R u_{*}\right)\right]^{2}\right\} \\
& N_{S, R}^{1}\left(u_{n_{l}}, u_{*}\right)=\min \left\{b_{p}\left(S u_{n_{l}}, R u_{*}\right) b_{p}\left(S u_{*}, R u_{n_{l}}\right), p_{b}\left(S u_{n_{l}}, R u_{*}\right) p_{b}\left(S u_{*}, S u_{n_{l}}\right), p_{b}\left(S u_{*}, R u_{n_{l}}\right) p_{b}\left(R u_{*}, R u_{n_{l}}\right)\right\}
\end{aligned}
$$


Since $\lim _{l \rightarrow \infty} N_{S, R}^{1}\left(u_{n_{l}}, u_{*}\right)=0$ and $\lim _{l \rightarrow \infty}=p_{b}\left(S u_{n_{l}}, R u_{n_{l}}\right)$. $p_{b}\left(S u_{*}, R u_{*}\right)=0$ (by) letting $l \longrightarrow \infty$ in (50), we have

either $\left[p_{b}\left(S u_{*}, R u_{*}\right)\right]^{2}=0$ or $\lim _{l \rightarrow \infty}\left[p_{b}\left(S u_{n_{l}}, S u_{*}\right)\right]^{2}=0$.

(1) If $\left[p_{b}\left(S u_{*}, R u_{*}\right)\right]^{2}=0$, it follows that $S u_{*}=R u_{*}$.

(2) If $\lim _{l \rightarrow \infty}\left[p_{b}\left(S u_{n_{l}}, S u_{*}\right)\right]^{2}=0$, by $(p b)_{4}$

$$
\begin{aligned}
p_{b}\left(R u_{*}, S u_{*}\right) & \leq s\left[p_{b}\left(R u_{*}, S u_{n_{l}}\right)+p_{b}\left(S u_{n_{l}}, S u_{*}\right)\right]-p_{b}\left(S u_{n_{l}}, S u_{n_{l}}\right) \\
& \leq s\left[p_{b}\left(R u_{*}, S u_{n_{l}}\right)+p_{b}\left(S u_{n_{l}}, S u_{*}\right)\right] \longrightarrow 0 \text { as } l \longrightarrow \infty,
\end{aligned}
$$

so $p_{b}\left(R u_{*}, S u_{*}\right)=0$.

Thereupon, $R u_{*}=S u_{*}=z$ and $z$ is a point of coincidence of $R$ and $S$.

Example 3. Let $M=\left\{\alpha_{1}, \alpha_{2}, \alpha_{3}, \alpha_{4}, \alpha_{5}\right\}$ and the partial $b$ -metric $p_{b}: M \times M \longrightarrow[0,+\infty)$ defined as follows (Table 1 ).
Let the function $\beta: M \times M \longrightarrow[0,+\infty)$, with

$\beta(u, y)= \begin{cases}1, & \text { for }(u, y) \in\left\{\left(\alpha_{5}, \alpha_{3}\right),\left(\alpha_{3}, \alpha_{2}\right)\right\} \\ 2, & \text { for }(u, y)=\left(\alpha_{2}, \alpha_{2}\right) \\ 0, & \text { otherwise }\end{cases}$

and two mappings $S, R: M \longrightarrow M$ (Table 2).

First of all, we remark that

$$
\begin{aligned}
& \beta\left(\alpha_{5}, \alpha_{3}\right)=\beta\left(R \alpha_{2}, R \alpha_{5}\right)=1 \Longrightarrow \beta\left(S \alpha_{2}, S \alpha_{5}\right)=\beta\left(\alpha_{3}, \alpha_{2}\right)=1, \\
& \beta\left(\alpha_{3}, \alpha_{2}\right)=\beta\left(R \alpha_{5}, R \alpha_{4}\right)=1 \Longrightarrow \beta\left(S \alpha_{5}, S \alpha_{4}\right)=\beta\left(\alpha_{2}, \alpha_{2}\right)=2, \\
& \beta\left(\alpha_{2}, \alpha_{2}\right)=\beta\left(R \alpha_{4}, R \alpha_{4}\right)=2 \Longrightarrow \beta\left(S \alpha_{4}, S \alpha_{4}\right)=\beta\left(\alpha_{2}, \alpha_{2}\right)=2,
\end{aligned}
$$

which shows as that (b) holds. Also, it is easy to see that (a) and (c) are satisfied, so it remains to be verified (42). We distinguish two cases as follows:

(1) $(u, y)=\left(\alpha_{2}, \alpha_{5}\right)$

$$
\begin{gathered}
M_{S, R}^{1}\left(\alpha_{2}, \alpha_{5}\right)=\min \left\{\left[p_{b}\left(S \alpha_{2}, S \alpha_{5}\right)\right]^{2},\left[p_{b}\left(S \alpha_{5}, R \alpha_{5}\right)\right]^{2}\right\}=\min \left\{\left[p_{b}\left(\alpha_{3}, \alpha_{2}\right)\right]^{2},\left[p_{b} b\left(\alpha_{2}, \alpha_{3}\right)\right]^{2}\right\}=9, \\
N_{S, R}^{1}\left(\alpha_{2}, \alpha_{5}\right)=\min \left\{b_{p}\left(S \alpha_{2}, R \alpha_{5}\right) b_{p}\left(S \alpha_{5}, R \alpha_{2}\right), \cdots\right\}=\min \left\{b_{p}\left(\alpha_{3}, \alpha_{3}\right) b_{p}\left(\alpha_{2}, \alpha_{5}\right), \cdots\right\}=0, \\
p_{b}\left(S \alpha_{2}, R \alpha_{2}\right) p_{b}\left(S \alpha_{5}, R \alpha_{5}\right)=p_{b}\left(\alpha_{3}, \alpha_{5}\right) p_{b}\left(\alpha_{2}, \alpha_{3}\right)=22 \cdot 3=66 .
\end{gathered}
$$

(2) $(u, y)=\left(\alpha_{5}, \alpha_{4}\right)$

$$
\begin{gathered}
M_{S, R}^{1}\left(\alpha_{5}, \alpha_{4}\right)=\min \left\{\left[p_{b}\left(S \alpha_{5}, S \alpha_{4}\right)\right]^{2},\left[p_{b}\left(S \alpha_{4}, R \alpha_{4}\right)\right]^{2}\right\}=\min \left\{\left[p_{b}\left(\alpha_{2}, \alpha_{2}\right)\right]^{2},\left[p_{b}\left(\alpha_{2}, \alpha_{2}\right)\right]^{2}\right\}=1, \\
N_{S, R}^{1}\left(\alpha_{5}, \alpha_{4}\right)=\min \left\{b_{p}\left(S \alpha_{5}, R \alpha_{4}\right) b_{p}\left(S \alpha_{4}, R \alpha_{5}\right), \cdots\right\}=\min \left\{b_{p}\left(\alpha_{2}, \alpha_{2}\right) b_{p}\left(\alpha_{2}, \alpha_{3}\right), \cdots\right\}=0, \\
p_{b}\left(S \alpha_{5}, R \alpha_{5}\right) p_{b}\left(S \alpha_{4}, R \alpha_{4}\right)=p_{b}\left(\alpha_{2}, \alpha_{3}\right) p_{b}\left(\alpha_{2}, \alpha_{2}\right)=3 \cdot 1=3 .
\end{gathered}
$$

So, for any $\kappa \in(0,1)$, the inequality (42) holds. Therefore, the mappings $S, R$ have a point of coincidence, which is $z=\alpha_{2}$

Corollary 13. Let $\left(M, p_{b}, s \geq 1\right)$ be a complete $p_{b}$-ms and two mappings $R, S: M \rightarrow M$. Suppose that there exist $\kappa \in(0,1)$ and $a>0$ such that

$$
M_{S, R}^{1}(u, y)-a \cdot N_{S, R}^{1}(u, y) \leq \kappa p_{b}(S u, R u) p_{b}(S y, R y),
$$

where

$$
\begin{aligned}
& M_{S, R}^{1}(u, y)=\min \left\{\left[p_{b}(S u, S y)\right]^{2},\left[p_{b}(S y, R y)\right]^{22}\right\}, \\
& N_{S, R}^{1}(u, y)=\min \left\{b_{p}(S u, R y) b_{p}(S y, R u), p_{b}(S u, R y) p_{b}(S u, S y), p_{b}(S y, R u) p_{b}(R u, R y)\right\},
\end{aligned}
$$


TABLE 1

\begin{tabular}{lccccc}
\hline$p_{b}(u, y)$ & $\alpha_{1}$ & $\alpha_{2}$ & $\alpha_{3}$ & $\alpha_{4}$ & $\alpha_{5}$ \\
\hline$\alpha_{1}$ & 0 & 2 & 6 & 30 & 42 \\
$\alpha_{2}$ & 2 & 1 & 3 & 21 & 31 \\
$\alpha_{3}$ & 6 & 3 & 2 & 14 & 22 \\
$\alpha_{4}$ & 30 & 21 & 14 & 5 & 7 \\
$\alpha_{5}$ & 42 & 31 & 22 & 7 & 6 \\
\hline
\end{tabular}

TABLE 2

\begin{tabular}{cccccc}
\hline & $\alpha_{1}$ & $\alpha_{2}$ & $\alpha_{3}$ & $\alpha_{4}$ & $\alpha_{5}$ \\
\hline$S$ & $\alpha_{5}$ & $\alpha_{3}$ & $\alpha_{2}$ & $\alpha_{2}$ & $\alpha_{2}$ \\
$R$ & $\alpha_{1}$ & $\alpha_{5}$ & $\alpha_{1}$ & $\alpha_{2}$ & $\alpha_{3}$ \\
\hline
\end{tabular}

for every $u, y \in M$, such that $u \neq y$ when $u, y \in C_{c}(R, S)_{M}$. Then, the mappings $S$ and $R$ have a point of coincidence providing that $S(M) \subset R(M)$ and $\left(R(M), p_{b}, s\right)$ is a $0-p_{b}$-complete $p_{b}$-ms.

Proof. Put $\beta(u, y)=1$ in Theorem 12 .

Theorem 14. Let $\left(M, p_{b}, s \geq 1\right)$ be a complete $p_{b}$-ms a mapping $S: M \longrightarrow M$. Suppose that there exists $\kappa \in(0,1)$ and $a>0$ such that

$$
\beta(u, y) M_{S}^{1}(u, y)-a \cdot N_{S}^{1}(u, y) \leq \kappa p_{b}(S u, u) p_{b}(S y, y),
$$

where

$M_{S}^{1}(u, y)=\min \left\{\left[p_{b}(S u, S y)\right]^{2},\left[p_{b}(S y, y)\right]^{2}\right\}$,

$N_{S}^{1}(u, y)=\min \left\{b_{p}(S u, y) b_{p}(S y, u), p_{b}(S u, y) p_{b}(S u, S y), p_{b}(S y, u) p_{b}(u, y)\right\}$,

for every $u, y \in M, u \neq y$. Suppose also that

(a) $S$ is $\beta$-admissible, and there exists $u_{0} \in M$ such that $\beta$ $\left(u_{0}, S u_{0}\right) \geq 1$

(b) $M$ is $\beta$-regular

Then, the mapping $S$ possesses a fixed point.

Proof. Choose $R=I_{M}$ in Theorem 12.

Corollary 15. Let $\left(M, p_{b}, s \geq 1\right)$ be a complete $p_{b}$-ms a mapping $S: M \longrightarrow M$. Suppose that there exists $\kappa \in(0,1)$ and $a>0$ such that

$$
M_{S}^{1}(u, y)-a \cdot N_{S}^{1}(u, y) \leq \kappa p_{b}(S u, u) p_{b}(S y, y),
$$

where
$M_{S}^{1}(u, y)=\min \left\{\left[p_{b}(S u, S y)\right]^{2},\left[p_{b}(S y, y)\right]^{2}\right\}$,

$N_{S}^{1}(u, y)=\min \left\{b_{p}(S u, y) b_{p}(S y, u), p_{b}(S u, y) p_{b}(S u, S y), p_{b}(S y, u) p_{b}(u, y)\right\}$,

for every $u, y \in M, u \neq y$. Then, the mapping $S$ possesses a fixed point.

Proof. Put $\beta(u, y)=1$ in Theorem 14.

Theorem 16. Let $\left(M, p_{b}, s \geq 1\right)$ be a complete $p_{b}$-ms and two mappings $R, S: M \longrightarrow M$. Suppose that there exist $\kappa \in(0,1)$ and $a>0$ such that

$$
\beta(R u, R y) M_{S, R}^{2}(u, y) \leq \kappa \cdot N_{S, R}^{2}(u, y),
$$

where

$M_{S, R}^{2}(u, y)=p_{b}(S u, S y) p_{b}(S y, R y)-a \cdot \min \left\{b_{p}(S u, R y), b_{p}(S y, R u)\right\}$,

$N_{S, R}^{2}(u, y)=p_{b}(R u, R y) \cdot \max \left\{p_{b}(S u, R u), p_{b}(S y, R y), \frac{p_{b}(S u, R y)+p_{b}(S y, R u)}{2 s}\right\}$

for every $u, y \in M$, such that $u \neq y$ when $u, y \in C_{c}(R, S)_{M}$. Suppose also that

(a) $S(M) \subset R(M)$ and $\left(R(M), p_{b}, s\right)$ is a $0-p_{b}$-complete $p_{b}$-ms

(b) $S$ is $R$ - $\beta$-admissible and there exists $u_{0} \in M$ such that $\beta\left(R u_{0}, S u_{0}\right) \geq 1$

(c) $M$ is $\beta$-regular

Then, the mappings $S$ and $R$ have a point of coincidence.

Proof. We will only sketch the proof, because, basically, we use the same technique that was used in the above theorems. Indeed, for $u=u_{n}, y=u_{n+1}$, where the sequences $\left\{z_{n}\right\},\left\{\mathrm{u}_{n}\right\}$ are defined in Theorem 8, we have

$$
\begin{aligned}
M_{S, R}^{2}\left(u_{n}, u_{n+1}\right)= & p_{b}\left(S u_{n}, S u_{n+1}\right) p_{b}\left(S u_{n+1}, R u_{n+1}\right) \\
& --a \cdot \min \left\{b_{p}\left(S u_{n}, R u_{n+1}\right), b_{p}\left(S u_{n+1}, R u_{n}\right)\right\} \\
= & p_{b}\left(z_{n}, z_{n+1}\right) p_{b}\left(z_{n+1}, z_{n}\right) \\
& -a \cdot \min \left\{b_{p}\left(z_{n}, z_{n}\right), b_{p}\left(z_{n+1}, z_{n-1}\right)\right\} \\
= & {\left[p_{b}\left(z_{n}, z_{n+1}\right)\right]^{2}, }
\end{aligned}
$$




$$
\begin{aligned}
& N_{S, R}^{2}\left(u_{n}, u_{n+1}\right)=p_{b}\left(R u_{n}, R u_{n+1}\right) \cdot \max \left\{\begin{array}{l}
p_{b}\left(S u_{n}, R u_{n}\right), p_{b}\left(S u_{n+1}, R u_{n+1}\right), \\
\frac{p_{b}\left(S u_{n}, R u_{n+1}\right)+p_{b}\left(S u_{n+1}, R u_{n}\right)}{2 s}
\end{array}\right\} \\
& =\max \left\{\begin{array}{l}
p_{b}\left(z_{n-1}, z_{n}\right), p_{b}\left(z_{n}, z_{n+1}\right), \\
\frac{p_{b}\left(z_{n}, z_{n}\right)+p_{b}\left(z_{n+1}, z_{n-1}\right)}{2 s}
\end{array}\right\} \\
& \leq p_{b}\left(z_{n-1}, z_{n}\right) \cdot \mathrm{m} p_{b}\left(z_{n-1}, z_{n}\right) \cdot \max \\
& \left\{\begin{array}{c}
p_{b}\left(z_{n-1}, z_{n}\right), p_{b}\left(z_{n}, z_{n+1}\right), \\
\frac{p_{b}\left(z_{n}, z_{n}\right)+s\left[p_{b}\left(z_{n+1}, z_{n}\right)+p_{b}\left(z_{n}, z_{n+1}\right)\right]-p_{b}\left(z_{n}, z_{n}\right)}{2 s}
\end{array}\right\} \\
& =p_{b}\left(z_{n-1}, z_{n}\right) \cdot \max \\
& \cdot\left\{p_{b}\left(z_{n-1}, z_{n}\right), p_{b}\left(z_{n}, z_{n+1}\right), \frac{p_{b}\left(z_{n+1}, z_{n}\right)+p_{b}\left(z_{n}, z_{n+1}\right)}{2}\right\} \\
& =p_{b}\left(z_{n-1}, z_{n}\right) \cdot \max \left\{p_{b}\left(z_{n-1}, z_{n}\right), p_{b}\left(z_{n}, z_{n+1}\right)\right\} \text {. }
\end{aligned}
$$

Thus, the inequality (64) becomes

$\left[p_{b}\left(z_{n}, z_{n+1}\right)\right]^{2} \leq \kappa p_{b}\left(z_{n-1}, z_{n}\right) \cdot \max \left\{p_{b}\left(z_{n-1}, z_{n}\right), p_{b}\left(z_{n}, z_{n+1}\right)\right\}$.

Since for the case $\max \left\{p_{b}\left(z_{n-1}, z_{n}\right), p_{b}\left(z_{n}, z_{n+1}\right)\right\}=p_{b}\left(z_{n}\right.$, $\left.z_{n+1}\right)$ we get $\left[p_{b}\left(z_{n}, z_{n+1}\right)\right]^{2} \leq \kappa p_{b}\left(z_{n-1}, z_{n}\right) \cdot p_{b}\left(z_{n}, z_{n+1}\right)$, or $p_{b}\left(z_{n}, z_{n+1}\right) \leq \kappa p_{b}\left(z_{n-1}, z_{n}\right)<p_{b}\left(z_{n-1}, z_{n}\right)$, which is a contradiction, we conclude that $\max \left\{p_{b}\left(z_{n-1}, z_{n}\right), p_{b}\left(z_{n}, z_{n+1}\right)\right\}=$ $p_{b}\left(z_{n-1}, z_{n}\right)$ and then (67) becomes

$$
p_{b}\left(z_{n}, z_{n+1}\right) \leq \kappa p_{b}\left(z_{n-1}, z_{n}\right)
$$

for any $n \in \mathbb{N}$. Therefore, by Lemma L2A and using similar arguments as in Theorems 8 and 12, there exists $u_{*} \in M$ such that

$$
\lim _{n \rightarrow \infty} p_{b}\left(S u_{n}, R u_{*}\right)=\lim _{n \rightarrow \infty} p_{b}\left(R u_{n}, R u_{*}\right)=p_{b}\left(R u_{*}, R u_{*}\right)=0
$$

Finally, we claim that $S u_{*}=R u_{*}$. From the assumptions (c), there exists a subsequences $\left\{u_{n_{l}}\right\}$ of $\left\{u_{n}\right\}$ such that $\beta\left(u_{n_{l}}\right.$, $\left.u_{*}\right) \geq 1$. Thus, replacing $u$ by $u_{n_{l}}$ and $y$ by $u_{*}$, we get (as $l \longrightarrow \infty)$

$$
\begin{aligned}
\lim _{n \rightarrow \infty} M_{S, R}^{2}\left(u_{n_{l}}, u_{*}\right)= & \lim _{n \rightarrow \infty}\left[p_{b}\left(S u_{n_{l}}, S u_{*}\right) p_{b}\left(S u_{*}, R u_{*}\right)\right. \\
& \left.--a \cdot \min \left\{b_{p}\left(S u_{n_{l}}, R u_{*}\right), b_{p}\left(S u_{*}, R u_{n_{l}}\right)\right\}\right] \\
= & p_{b}\left(S u_{*}, R u_{*}\right) \cdot \lim _{n \rightarrow \infty}\left[p_{b}\left(S u_{n_{l}}, S u_{*}\right),\right. \\
\lim _{n \rightarrow \infty} N_{S, R}^{2}\left(u_{n_{l}}, u_{*}\right)= & \lim _{n \rightarrow \infty} p_{b}\left(R u_{n_{l}}, R u_{*}\right) \\
& \cdot \max \left\{\begin{array}{l}
p_{b}\left(S u_{n_{l}}, R u_{n_{l}}\right), p_{b}\left(S u_{*}, R u_{*}\right), \\
\left.\frac{p_{b}\left(S u_{n_{l}}, R u_{*}\right)+p_{b}\left(S u_{*}, R u_{n_{l}}\right)}{2 s}\right\}=0 .
\end{array}\right.
\end{aligned}
$$

Consequently, (64) becomes $p_{b}\left(S u_{*}, R u_{*}\right) \cdot \lim _{n \rightarrow \infty}\left[p_{b}\left(S u_{n_{l}}\right.\right.$,
$\left.S u_{*}\right)=0$ and the rest is just a verbatim repetition of the lines in the previous proofs.

Corollary 17. Let $\left(M, p_{b}, s \geq 1\right)$ be a complete $p_{b}$-ms and two mappings $R, S: M \longrightarrow M$. Suppose that there exist $\kappa \in(0,1)$ and $a>0$ such that

$$
M_{S, R}^{2}(u, y) \leq \kappa \cdot N_{S, R}^{2}(u, y)
$$

where

$M_{S, R}^{2}(u, y)=p_{b}(S u, S y) p_{b}(S y, R y)-a \cdot \min \left\{b_{p}(S u, R y), b_{p}(S y, R u)\right\}$,

$N_{S, R}^{2}(u, y)=p_{b}(R u, R y) \cdot \max \left\{p_{b}(S u, R u), p_{b}(S y, R y), \frac{p_{b}(S u, R y)+p_{b}(S y, R u)}{2 s}\right\}$,

for every $u, y \in M$, such that $u \neq y$ when $u, y \in C_{c}(R, S)_{M}$. If $S$ $(M) \subset R(M)$ and $\left(R(M), p_{b}, s\right)$ is a $0-p_{b}$-complete $p_{b}-m s$, then, the mappings $S$ and $R$ have a point of coincidence.

Proof. Let $\beta(u, y)=1$ in Theorem 16 .

Theorem 18. Let $\left(M, p_{b}, s \geq 1\right)$ be a complete $p_{b}$-ms and $a$ mapping $S: M \longrightarrow M$. Suppose that there exist $\kappa \in(0,1)$ and $a>0$ such that

$$
\beta(u, y) M_{S}^{2}(u, y) \leq \kappa \cdot N_{S}^{2}(u, y)
$$

where

$M_{S}^{2}(u, y)=p_{b}(S u, S y) p_{b}(S y, y)-a \cdot \min \left\{b_{p}(S u, y), b_{p}(S y, u)\right\}$,

$N_{S}^{2}(u, y)=p_{b}(u, y) \cdot \max \left\{p_{b}(S u, u), p_{b}(S y, y), \frac{p_{b}(S u, y)+p_{b}(S y, u)}{2 s}\right\}$,

for every $u, y \in M$. Suppose also that

(i) $S$ is $\beta$-admissible, and there exists $u_{0} \in M$ such that $\beta\left(R u_{0}, S u_{0}\right) \geq 1$

(ii) $M$ is $\beta$-regular

Then, the mapping S admits a fixed point.

Proof. Choose $R=I_{M}$.

Corollary 19. Let $\left(M, p_{b}, s \geq 1\right)$ be a complete $p_{b}$-ms and two mappings $R, S: M \longrightarrow M$. Suppose that there exist $\kappa \in(0,1)$ and $a>0$ such that

$$
M_{S}^{2}(u, y) \leq \kappa \cdot N_{S}^{2}(u, y)
$$

where 


$$
\begin{aligned}
& M_{S}^{2}(u, y) M_{S}^{2}(u, y)=p_{b}(S u, S y) p_{b}(S y, y)-a \cdot \min \left\{b_{p}(S u, y), b_{p}(S y, u)\right\}, \\
& N_{S}^{2}(u, y)=p_{b}(u, y) \cdot \max \left\{p_{b}(S u, u), p_{b}(S y, y), \frac{p_{b}(S u, y)+p_{b}(S y, u)}{2 s}\right\},
\end{aligned}
$$

for every $u, y \in M$. Then, the mapping $S$ has a fixed point.

\section{Data Availability}

No data were used to support this study.

\section{Conflicts of Interest}

The authors declare that they have no competing interests.

\section{Authors' Contributions}

Both authors contributed equally and significantly in writing this paper. Both authors read and approved the final manuscript.

\section{Acknowledgments}

This research was supported by the Ministry of Science and Technology of the Republic of China.

\section{References}

[1] L. B. Ćirić, "On some maps with a non-unique fixed point," Publications de l'Institut Mathématique, vol. 17, pp. 52-58, 1974.

[2] E. Picard, "Memoire sur la theorie des equations aux derivees partielles et la methode des approximations successives," Journal de Mathématiques Pures et Appliquées, vol. 6, pp. 145-210, 1890.

[3] S. Gulyaz-Ozyurt, "A note on Kannan type mappings with a Fcontractive iterate," Results in Nonlinear Analysis, vol. 2, no. 3, pp. 143-146, 2019.

[4] C.-M. Chen and C.-C. Kuo, "Best proximity point theorems of cyclic Meir-Keeler-Kannan-Chatterjea contractions," Results in Nonlinear Analysis, vol. 2, no. 2, pp. 83-91, 2019.

[5] I. A. Rus, "Some variants of contraction principle in the case of operators with Volterra property: step by step contraction principle," Advances in the Theory of Nonlinear Analysis and its Applications, vol. 3, no. 3, pp. 111-120, 2019.

[6] J. Achari, "On Ćirić's non-unique fixed points," Matematichki Vesnik, vol. 13, pp. 255-257, 1976.

[7] B. G. Pachpatte, "On Ćirić type maps with a non-unique fixed point," Indian Journal of Pure and Applied Mathematics, vol. 10, pp. 1039-1043, 1979.

[8] L. Ćirić and N. Jotić, "A further extension of maps with nonunique fixed points," Matematički Vesnik, vol. 50, pp. 1-4, 1998.

[9] E. Karapinar, “A new non-unique fixed point theorem," Journal of Functional Analysis, vol. 7, no. 1-2, pp. 92-97, 2012.

[10] E. Karapınar and R. P. Agarwal, "A note on Ćirić type nonunique fixed point theorems," Fixed Point Theory and Applications, vol. 2017, no. 1, Article ID 20, 2017.
[11] F. Zhang, S. M. Kang, and L. Xie, "Ćirićs type mappings with a non-unique coincidence points," Fixed Point Theory and Applications, vol. 6, 190 pages, 2007.

[12] J. Zhou, D. Zheng, and G. Zhang, "Fixed point theorems in partial b-metric spaces," Applied Mathematical Sciences, vol. 12, no. 13, pp. 617-624, 2018.

[13] J. Vujaković, H. Aydi, S. Radenović, and A. Mukheimer, "Some remarks and new results in ordered partial $b$-metric spaces," Mathematics, vol. 7, no. 4, p. 334, 2019.

[14] E. Karapinar, "A short survey on the recent fixed point results on b-metric spaces," Constructive Mathematical Analysis, vol. 1, no. 1, pp. 15-44, 2018.

[15] B. Alqahtani, A. Fulga, and E. Karapınar, "Non-unique fixed point results in extended B-metric space," Mathematics, vol. 6, no. 5, p. 68, 2018.

[16] H. H. Alsulami, E. Karapınar, and V. Rakocevic, "Ćirić type nonunique fixed point theorems on b-metric spaces," Filomat, vol. 31, no. 11, pp. 3147-3156, 2017.

[17] H. Aydi, E. Karapınar, and V. Rakoçević, "Nonunique fixed point theorems on $b$-metric spaces via simulation functions," Jordan Journal of Mathematics and Statistics, vol. 12, no. 3, pp. 265-288, 2019.

[18] Z. Kadelburg and S. Radenović, "Notes on some recent papers concerning $F$-contractions in $b$-metric spaces," Constructive Mathematical Analysis, vol. 1, no. 2, pp. 108-112, 2018.

[19] E. Karapınar, "Ćirić types non-unique fixed point results: a review," Applied and Computational Mathematics, vol. 1, pp. 3-21, 2019.

[20] A. S. Babu, "Some Presic type results in $b$ dislocated metric spaces," Constructive Mathematical Analysis, vol. 2, no. 1, pp. 40-48, 2019.

[21] E. Karapınar, "Ćirić types nonunique fixed point theorems on partial metric spaces," Journal of Nonlinear Sciences and Applications, vol. 5, no. 2, pp. 74-83, 2012.

[22] E. Karapinar and S. Romaguera, "Nonunique fixed point theorems in partial metric spaces," Univerzitet u Nišu, vol. 27, no. 7, pp. 1305-1314, 2013.

[23] O. Acar, I. Altun, and S. Romaguera, "Caristi's type mappings on complete partial metric spaces," Fixed Point Theory, vol. 14, no. 1, pp. 3-10, 2013.

[24] E. Karapınar, "Some nonunique fixed point theorems of Ćirić type on cone metric spaces," Abstract and Applied Analysis, vol. 2010, Article ID 123094, 14 pages, 2010.

[25] S. Shukla, "Partial $b$-metric spaces and fixed point theorems," Mediterranean Journal of Mathematics, vol. 11, no. 2, pp. 703-711, 2014.

[26] N. V. Dung and V. T. L. Hang, "Remarks on partial $b$-metric spaces and fixed point theorems," Matematicki Vesnik, vol. 69, pp. 231-240, 2017.

[27] Z. Mustafa, J. Roshan, V. Parvaneh, and Z. Kadelburg, "Some common fixed point results in ordered partial $b$-metric spaces," Journal of Inequalities and Applications, vol. 2013, no. 1, Article ID 562, 2013.

[28] V. La Rosa and P. Vetro, "Common fixed points for $\alpha-\psi-\varphi$ -contractions in generalized metric spaces," Nonlinear Analysis: Modelling and Control, vol. 19, no. 1, pp. 43-54, 2014.

[29] Z. D. Mitrović, "A note on the results of Suzuki, Miculescu and Mihail," Journal of Fixed Point Theory and Applications, vol. 21, no. 1, p. 24, 2019. 